\title{
Preparation, Characterization, and Determination of Immunological Activities of Transfer Factor Specific to Human Sperm Antigen
}

\author{
Jianwei Zhou, ${ }^{1}$ Cui Kong, ${ }^{1}$ Zhaohong Yuan, ${ }^{1}$ Junmin Luo, ${ }^{2}$ Rui Ma, ${ }^{2}$ Jiang Yu, ${ }^{1}$ and Jinghe Cao ${ }^{3}$ \\ ${ }^{1}$ Clinical Laboratory, The Affiliated Hospital of Jining Medical College, Shandong, Jining 272029, China \\ ${ }^{2}$ Department of Immunology, Zunyi Medical College, Guizhou, Zunyi 563003, China \\ ${ }^{3}$ Medical Reproduction Centre, The Affiliated Hospital of Jining Medical College, Shandong, Jining 272029, China \\ Correspondence should be addressed to Jianwei Zhou; immunolife@126.com
}

Received 16 August 2012; Revised 22 October 2012; Accepted 7 November 2012

Academic Editor: Andre Van Wijnen

Copyright (C) 2013 Jianwei Zhou et al. This is an open access article distributed under the Creative Commons Attribution License, which permits unrestricted use, distribution, and reproduction in any medium, provided the original work is properly cited.

\begin{abstract}
Objective. The objective of this study was to prepare, characterize, and determine immunological activities of specific transfer factor (STF) specific to human sperm antigen (HSA) for the preparation of antisperm contraceptive vaccine that can be used as an immunocontraceptive. Methods. HSA-STF was prepared using the spleens of rabbits vaccinated with HSA. The specific immunological activities were examined by lymphocyte proliferation test (LPT), leukocyte adhesion inhibition test (LAIT), and by determining the concentrations of IL-4, $\gamma$-IFN, and IL-21. HSA-STF was a helveolous substance, having a $\mathrm{pH}$ value of $7.0 \pm 0.4$ and UV absorption maxima at $258 \pm 6 \mathrm{~nm}$. It contained seventeen amino acids; glycine and glutamic acids were the highest in terms of concentrations $(38.8 \mu \mathrm{g} / \mathrm{mL}$ and $36.3 \mu \mathrm{g} / \mathrm{mL}$, resp.). Results. The concentration of polypeptide was $2.34 \pm 0.31 \mathrm{mg} / \mathrm{mL}$, and ribose was $0.717 \pm 0.043 \mathrm{mg} / \mathrm{mL}$. The stimulation index for lymphocyte proliferation test was 1.84 , and the leukocyte adhesion inhibition rate was $37.7 \%$. There was a statistically significant difference between the cultural lymphocytes with HSA-STF and non-HSA-STF for $\gamma$-IFN and IL-21 $(P<0.05)$, but there was no statistical significance for IL-4 $(P>0.05)$. Conclusion. HSA-STF was prepared and characterized successfully. It had immunological activity which could transfer the immune response specific to HSA and prove to be a potential candidate for the development of male immunocontraceptive agents.
\end{abstract}

\section{Introduction}

The burgeoning population has major implications worldwide. In the face of this problem, birth control was regarded as important by many countries. Contraception is the key measure for birth control, but traditional measures have been misused in many ways; for example, easy desquamation of intrauterine ring, possible injury to person with hypodesmus, side effects of contraceptive, and condom effects. Currently available contraceptive methods are not well suited to the religious, social, economic, or health circumstances worldwide. So, there is an urgent need to develop harmless, retrievable products for contraception. Reproductive science has provided a range of fertility control measures for women, but the choices for men are few and currently limited to condoms and vasectomy [1]. Various health organizations and pharmaceutical companies actively continue to pursue research towards new contraceptive approaches [2]. Presently, based on the variable antigens (Ags) existing in the male reproductive system, researchers have developed many antibodies (Abs) or vaccines for contraception, such as Abs to SP-10 expressed in the testis that could interfere with the union of sperm and ovum [3]. The sperm-specific protein, Izumo, a member of immunoglobulin superfamily (IgSF) which is located on the sperm stimulates hamster to secrete high titer of Abs both in the genital tract and serum and further decreases their reproductive capability [4]. Antibodies to Eppin (epididymal protease inhibitor), a newly found protein secreted by testis and epididymis, could itself act as a candidate vaccine because of its ability to regulate the liquefaction of semen and limit sperm motility $[5,6]$. There are several evident shortcomings regardless of the type 
TABLE 1: Schedule for inoculating rabbits with immunogen (HSA).

\begin{tabular}{lcccc}
\hline Week & CFA $(\mathrm{mL})$ & IFA $(\mathrm{mL})$ & Immunogen $(\mathrm{mL})$ & Inoculation pathway \\
\hline 1st & 0.5 & 0.5 & 1 & Back, abdomen, neck, armpit \\
2nd & - & - & 2 & Back, abdomen, neck, armpit \\
3th & - & - & 2 & Back, abdomen, neck, armpit \\
4th & - & - & 4 & Back, abdomen, neck, armpit \\
5th & - & - & 4 & Back, abdomen, neck, armpit \\
\hline
\end{tabular}

“-” represents this component that was not present in the immunogenic mixture.

of vaccination, such as weak antigenicity and short persistence time. Hence, it is extremely necessary to prepare an adjuvant to stimulate the immune system and enhance the immune response to Ags, especially, to pregnancy vaccines.

Specific transfer factor (STF) is low molecular weight peptides composed of number of amino acid residues and capable of transferring immunity from one cell or individual to another. It is an immunoregulatory and immunosupportive agent with normalizing effect on abnormal immune response. It is prepared by the spleen or peripheral blood lymphocytes of the animals inoculated with a certain pathogen. The essential components of STF are polypeptides and nucleotides. One of the unique features of STF is that it does not have immunogenicity and genus specificity but the STF prepared by one genus of animals like goat, rabbit, dog, and so forth has the ability of transferring the immunological activity to other genera of animals without causing hypersensitivity. Currently, several STFs have been successfully developed; mainly for herpes simplex virus type 1 (HSV1) [7], Epstein-Barr virus [8], and Staphylococcus aureus antigens [9]. Also, STFs have been used in therapy of many diseases, for example, lyme, candidiasis, and herpes simplex [10-14]. However, till date no literature has been reported about the preparation of specific transfer factor related to pregnancy vaccine.

In context with the previous work on transfer factors [15-17], the present study focused on preparation, characterization, and determination of immunological activities of transfer factor specific to human sperm antigen. We anticipate that the findings from this study would provide valuable information for developing high-efficiency vaccines for birth control in China.

\section{Materials and Methods}

2.1. Animals, Instruments and Reagents. Rabbits and cony pigs were bought from the Experimental Animal Centre of Shandong University, China. All the animals were utilized by the rules related to experimental animals in China. The instruments utilized for the study were ultraspectrophotometer (model number 6405, JENWAY), tissue disintegrator (Fuhua of Jiangsu), Elx-800 microplate-reader (Bio-TEK), and Hitachi 835-50 analyzer. The reagents used included phytohemagglutinin (PHA), methyl thiazolyl tetrazolium (MTT), and D ribose. All the reagents were purchased from Sigma Ltd. Cytokine ELISA kit was purchased from BD Ltd.
2.2. Preparation of Immunogen (HSA Solution). Sperm samples were washed five times with sterile saline and diluted with Roswell Park Memorial Institute (RPMI) 1640 medium. After the sperm suspension concentration was adjusted to $4.0 \times 10^{6}$ sperm cells $/ \mathrm{mL}$, the suspension was completely subjected to comminution by Ultrasonic Cell Crusher maintained at $-4^{\circ} \mathrm{C}$. The human sperm antigen (HSA) solution was thus prepared.

2.3. Immunization of Rabbits. For the initial immunization, the rabbits were injected subcutaneously with HSA solution mixed with equivalent volumes of complete Freund's adjuvant (CFA) and incomplete Freund's adjuvant (IFA). The response to immunization with HSA was considered successful if the titer of anti-HSA was $1: 1280$. The inoculation schedule for rabbits is presented in Table 1.

2.4. Preparation of HSA-STF. Ten adult, male rabbits weighing 2-3 kg were bought from Experimental Animal Centre of Jining Medical College, China. The rabbits vaccinated with HSA were successfully slaughtered, and the spleens were collected after cutting off vessels and fascia. The spleens were washed with sterile saline and mechanically crushed using high-speed tissue disintegrator and, subsequently, comminuted by Ultrasonic Cell Crusher under low temperature. Following this procedure, the spleen suspension thus obtained was frozen at $-20^{\circ} \mathrm{C}$ and thawed at $37^{\circ} \mathrm{C}$. This procedure was repeated six times. The liquid was dialyzed for 48 hours using dialysis tubing having molecular weight cutoff of 5,000 dalton. The dialysate was collected after aseptic filtration and stored in a refrigerator at $-20^{\circ} \mathrm{C}$ for further examination.

2.5. Analysis of General Physicochemical Properties. In accordance with the standard of transfer factor published in Chinese Pharmacopoeia (2005 Edition), the properties of HSASTF, including color, $\mathrm{pH}$, absorption peak, content of polypeptides and ribose, sterility test, pyrogen test, and safety test were determined. The content of polypeptide and ribose was detected by Orcinol assay and modified Lowry assay, respectively. Sterility test, pyrogen test, and safety test were carried out using traditional methods.

2.6. Analysis of Ultraviolet Spectrum and Amino Acids. The absorption peaks of HSA-STF solution were scanned in full wavelength using ultraspectrophotometer to find out the maximum absorption wavelength, and the ratio of $E_{260} / E_{280}$ 
was recorded. Content of amino acids in HSA-STF was detected using Hitachi 835-50 analyzer.

2.7. Determination of Immunological Activity. Venous blood was drawn from 20 healthy donors, and peripheral blood mononuclear cells (PBMCs) were isolated. The cells were washed twice with RPMI 1640 medium. The percentage of living cells was determined by Taipan blue staining. If the ratio was more than $95 \%$, then, according to the experimental need, various concentrations of cell suspension were prepared with RPMI 1640 medium containing 20\% fetal bovine serum (FBS). The specific immune activity was analyzed by lymphocyte proliferation test (LPT), leukocyte adhesion inhibition test (LAIT), and by detecting T-cell expansion.

2.7.1. Lymphocyte Proliferation Test (LPT). Cell suspension $(100 \mu \mathrm{L})$ with concentration $3 \times 10^{6} / \mathrm{mL}$ was placed into seven culture wells of 96-well plate. In the first six wells, $50 \mu \mathrm{L}$ PHA, $50 \mu \mathrm{L}$ HSA with optimal concentration $(4.0 \times$ $10^{6}$ sperm cells $\left./ \mathrm{mL}\right)$, and $50 \mu \mathrm{L}$ HSA-STF with gradient concentration (polypeptide concentration of HSA-STF $1 \mathrm{mg} /$ $\mathrm{mL} ; 0.25 \mathrm{mg} / \mathrm{mL} ; 0.063 \mathrm{mg} / \mathrm{mL} ; 0.016 \mathrm{mg} / \mathrm{mL} ; 0.004 \mathrm{mg} / \mathrm{mL}$; $0.001 \mathrm{mg} / \mathrm{mL}$ ) were added. The seventh well was treated as control to which only $100 \mu \mathrm{L}$ cultural solution was added. Each well was filled with RPMI 1640 medium containing $20 \%$ FBS, until the volume was $300 \mu \mathrm{L}$. Subsequently, the plate containing the cell suspensions and test substances were cultured at $37^{\circ} \mathrm{C}, 5 \% \mathrm{CO}_{2}$ atmosphere for 68 hours. After the incubation period, $20 \mu \mathrm{L}$ MTT and $200 \mu \mathrm{L}$ dimethyl sulfoxide (DMSO) were, respectively, added into each well. The final solution was used for measuring the optical density (OD) by enzyme-labeled meter using dual wavelength $(570 \mathrm{~nm}$ and $630 \mathrm{~nm}$ ). Stimulation index (SI) was calculated using the following formula:

$$
\mathrm{SI}=\frac{\mathrm{OD}_{\mathrm{Exp}}}{\mathrm{OD}_{\mathrm{Con}}}
$$

2.7.2. Leukocyte Adhesion Inhibition Test (LAIT). Cell suspension $(100 \mu \mathrm{L})$ with concentration $5 \times 10^{6} / \mathrm{mL}$ was placed into the first six experimental wells of 96 -well plate. Then, $50 \mu \mathrm{L}$ of HSA-STF with polypeptide concentration of $0.063 \mathrm{mg} / \mathrm{mL}$ was added. Cell suspension $(100 \mu \mathrm{L})$ plus $50 \mu \mathrm{L}$ RPMI 1640 medium containing 10\% FBS was added into the successive three wells treated as controls. These solutions were cultured for 2 hours at $37^{\circ} \mathrm{C}$ and $5 \% \mathrm{CO}_{2}$ atmosphere. After the incubation period, $50 \mu \mathrm{L}$ HSA with optimal concentration $\left(4.0 \times 10^{6}\right.$ sperm cells $\left./ \mathrm{mL}\right)$ and $50 \mu \mathrm{L}$ PHA solution were added to the first three experimental wells, and $50 \mu \mathrm{L}$ PHA plus RPMI 1640 medium containing 10\% FBS were added to the remaining six wells. The solutions in all the wells were again cultured for 2 hours. After decanting the supernatant fraction, $180 \mu \mathrm{L}$ RPMI $1640,20 \mu \mathrm{L}$ MTT, and $20 \mu \mathrm{L}$ DMSO were added to the remaining sperm cell fraction. The final solution was used for measuring the OD.
Leukocyte adhesion inhibition rate (LAIR) was calculated using the following formula:

$$
\operatorname{LAIR}=\left[\frac{\left(\mathrm{OD}_{\mathrm{Con}}-\mathrm{OD}_{\mathrm{Exp}}\right)}{\mathrm{OD}_{\mathrm{Con}}}\right] \times 100 \% .
$$

2.7.3. Detection of T-Cell Expansion. The procedure used for the preparation of cell culture was same as that for LAIT. The first three experimental wells were filled with $50 \mu \mathrm{L}$ HSA-STF having polypeptide concentration of $0.063 \mathrm{mg} / \mathrm{mL}$. In the middle three wells (control 1) and the subsequent three wells (control 2), $50 \mu \mathrm{L}$ RPMI 1640 containing 10\% FBS was added. These solutions were cultured for 2 hours at $37^{\circ} \mathrm{C}, 5 \% \mathrm{CO}_{2}$ atmosphere. After the incubation period, $50 \mu \mathrm{L}$ HSA was added into the first six wells, and $50 \mu \mathrm{L}$ RPMI 1640 containing $10 \% \mathrm{FBS}$ was added into the remaining three wells, and again cultured for 2 hours. Finally, the supernatant was carefully collected, and the concentration of IL-4, $\gamma$-IFN, and IL-21 was detected using ELISA kit.

2.7.4. The Contents for Transfer Assays Assessed by Skin Tests. $30 \mathrm{BALB} / \mathrm{c}$ mice, each weigh about $20 \mathrm{~g}$, were taken as the experimental animals, and they were divided into STF, NTF (normal transfer factor, prepared by the spleen of healthy rabbits), and control group randomly, the mice of the group were given HSA-STF, NTF, and saline water, respectively, both the concentrations of HSA-STF and NTF were same as $0.63 \mathrm{mg} / \mathrm{mL}$, and all the doses were $0.5 \mathrm{~mL}$ every day. On the 8th day, the pouring was stopped, and all were injected intracutaneously with HSA $0.5 \mathrm{~mL}$ and observed if there were any nodules formed on the skin.

2.8. Statistical Analysis. All data were analyzed with SPSS 15.0 software. Quantitative data was presented as $\bar{x} \pm s$ (mean \pm $\mathrm{SD}$ ). Single elemental data analysis was performed using chisquare test whereas $t$-test was used for comparing paired sample sets. Statistical significance was at $P \leq 0.05$.

\section{Results}

3.1. General Physicochemical Properties of HSA-STF. The HSA-STF was a helveolous substance with $\mathrm{pH}$ value of $7.0 \pm 0.4$. The HSA-STF had polypeptide content of $2.34 \pm$ $0.31 \mathrm{mg} / \mathrm{mL}$ and ribose up to $0.717 \pm 0.043 \mathrm{mg} / \mathrm{mL}$ (Table 2). In accordance with the standards of Chinese Pharmacopeia (2005 Edition), the results of protein qualitative test, sterility test, pyrogen test, and safety test were all negative. The preparation contained 17 amino acid residues in varying concentrations, out of which glycine (Gly) and glutamic acid (Glu) were the key amino acids present in the highest amount, followed by lysine (Lys) and alanine (Ala). The amounts of threonine (Thr), methionine (Met), and cystine (Cys) were the lowest (Table 3).

3.2. Analysis of Ultraviolet Spectrum of HSA-STF. Scanned in the range between $200 \mathrm{~nm}$ and $450 \mathrm{~nm}$ with ultraspectrophotometer, HSA-STF had a maximum absorption peak 
TABLE 2: Physicochemical properties of HSA-STF.

\begin{tabular}{lc}
\hline Tests & HSA-STF \\
\hline Color & Helveolous \\
Protein qualitative test & Negative \\
Sterile test & Negative \\
Safety test & Negative \\
Pyrogen test & Negative* \\
$\mathrm{pH}$ & $7.0 \pm 0.4$ \\
$E_{260} / E_{280}$ & $1.96 \pm 0.23$ \\
Maximum absorbance & $258 \pm 6$ \\
Concentration of polypeptide $(\mathrm{mg} / \mathrm{mL})$ & $2.34 \pm 0.31$ \\
Concentration of ribose $(\mathrm{mg} / \mathrm{mL})$ & $0.717 \pm 0.043$ \\
\hline
\end{tabular}

* It qualifies as per Chinese Pharmacopoeia.

TABLE 3: Concentration of amino acids in HSA-STF $(\mu \mathrm{g} / \mathrm{mL})$.

\begin{tabular}{lc}
\hline Amino acids & Concentration \\
\hline Arg & 11.4 \\
Lys & 33.1 \\
Ala & 22.7 \\
Thr & 3.6 \\
Gly & 38.8 \\
Val & 13.7 \\
Ser & 8.8 \\
Pro & 9.9 \\
Ile & 8.4 \\
Leu & 16.5 \\
Met & 3.4 \\
His & 7.9 \\
Phe & 11.6 \\
Glu & 36.3 \\
Asp & 7.9 \\
Cys & 2.2 \\
Tyr & 13.1 \\
\hline
\end{tabular}

at $252 \sim 262 \mathrm{~nm}$ as depicted in Figure 1. The $E_{260} / E_{280}$ was $1.96 \pm 0.23$.

3.3. Lymphocyte Proliferation Test (LPT). The proliferation of lymphocytes in the experimental wells containing HSASTF was significant as compared to the control. HSA-STF could enhance the proliferation of lymphocytes significantly. Moreover, the effect was dependent on the concentration of HSA-STF. The stimulation index (SI) for lymphocyte proliferation test was 1.84 when the polypeptide concentration was $0.063 \mathrm{mg} / \mathrm{mL}$ (Figure 2).

3.4. Leukocyte Adhesion Inhibition Test (LAIT). After incubation with HSA-STF, the LAIR of the lymphocytes in the experimental wells containing HSA plus PHA was $37.7 \%$, which was significantly higher than other wells containing either HSA or PHA $(P<0.05)$ (Figure 3). This finding suggested that HSA-STF could inhibit leukocyte adhesion, and the effect of LAI was dependent on specific antigen.

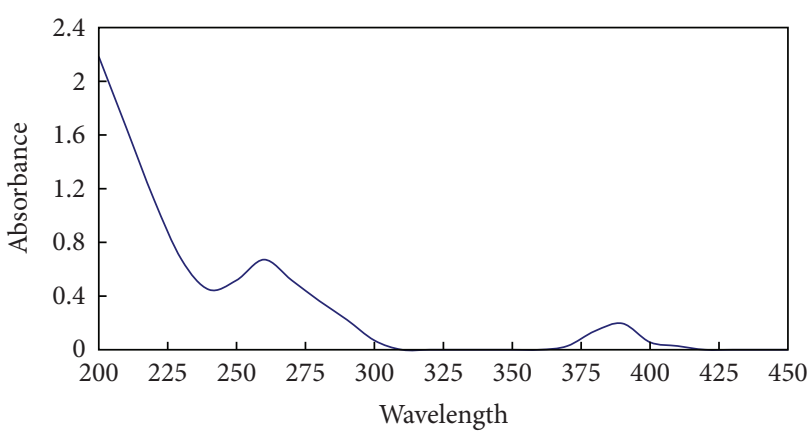

FIGURE 1: Multi wavelength scanning graph of HSA-STF.

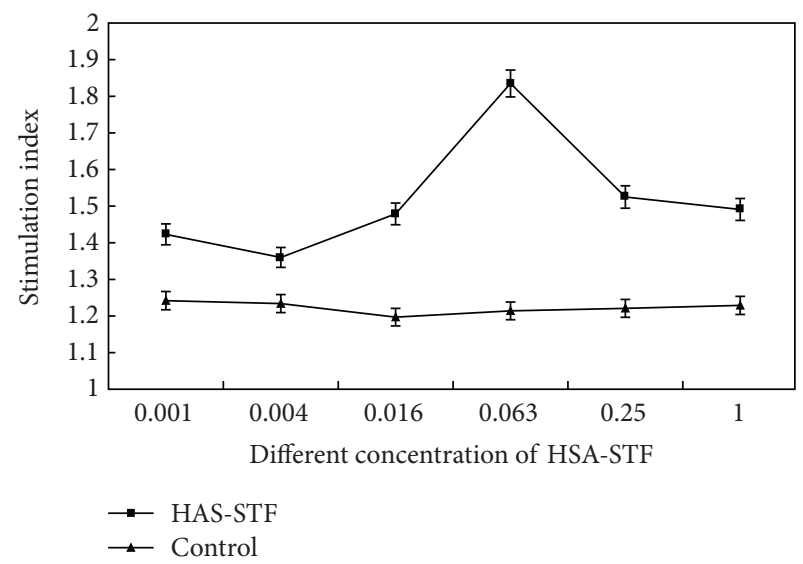

Figure 2: Effect of HSA-STF with different concentration of polypeptide on lymphocyte proliferation.

3.5. Expansion of T-Cell Subpopulation. Compared with the control-1 group, there was no significant difference $(P>$ 0.05 ) in IL-4 content for the experimental group, but there was a significant difference $(P<0.05)$ between the two groups for the levels of $\gamma$-IFN and IL-21. Similar results were observed for the experimental group and control-2 group (Table 4).

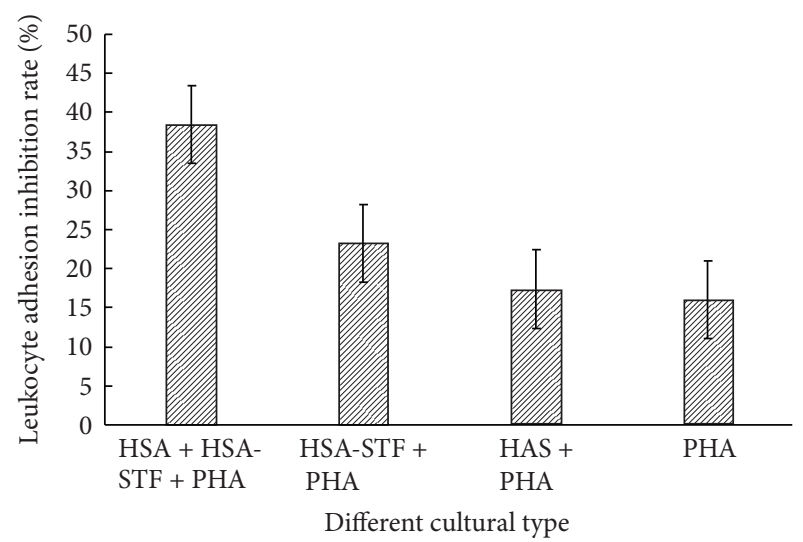

FIGURE 3: Leukocyte adhesion inhibition rate of HSA-STF. 
TABLE 4: Concentration of cytokines secreted by lymphocytes under the function of HSA-STF $(\mathrm{pg} / \mathrm{mL})$.

\begin{tabular}{lccc}
\hline Cytokines & Experiment group & Control 1 & Control 2 \\
\hline IL-4 & $234 \pm 84$ & $226 \pm 51$ & $209 \pm 77$ \\
$\gamma$-IFN & $595 \pm 118$ & $337 \pm 105^{*}$ & $268 \pm 99^{*}$ \\
IL-21 & $403 \pm 147$ & $214 \pm 88^{*}$ & $159 \pm 59^{*}$ \\
\hline
\end{tabular}

${ }^{*}$ There was a significant difference $(P<0.05)$ between experimental group and control group.

3.6. The Contents for Transfer Assays Assessed by Skin Tests. In the STF group, the skin was inflamed at the site of injection, and there were marked nodules formed, while there was no nodule observed in the mice in both NTF and control groups.

\section{Discussion}

The development of vaccines based on sperm antigen is an exciting proposition and may represent a promising alternative to the currently available contraceptive methods [4]. In this study, we prepared and characterized HSA-STF successfully, and the physicochemical properties (including the color, absorption peak, the content of polypeptide, and ribose) were determined. The immunological activity of HSA-STF was also determined. The HSA-STF acted in accordance with the standard of transfer factor published in Chinese Pharmacopeia (2005 Edition). There were conflicting reports for the number of amino acids present in HSASTF. Some reported 17 while others reported 18 amino acids $[18,19]$. Moreover, the content of each amino acid varied from study to study. On the contrary, a study by Kirkpatrick stated that multiple combinatorial patterns between these amino acids create a vast number of different transfer factor molecules. Such large number of molecules would then satisfy the notion that STF molecule was necessary to transfer immunity to each and every specific antigenic determinant [20].

Lymphocyte proliferation test is used to detect the nonspecific characteristic of STF. The results showed that HSA-STF stimulated the proliferation of lymphocytes, which resulted in increase in the concentration of polypeptide as increased in SI. When the concentration of polypeptide was more than $0.063 \mathrm{mg} / \mathrm{mL}$, the SI decreased. On the contrary, this phenomenon showed, that after stimulation by HSA-STF, the increase or decrease in lymphocytes was dependent upon the concentration of polypeptide. These results were in line with another study by Lawrence [21]. A study by Xiao et al. 2004 [22] reported that the optical concentration of TF specific to tuberculosis was $0.50 \mathrm{mg} / \mathrm{mL}$. This is probably due to the differences in HSA-STF components, which decide its stimulation activities. According to this characteristic, a study by Kirkpatrick et al. 1985 [23] was focused to prepare STF by immunizing mice with artificial amino acids. This resulted in successful transfer of the delayed type hypersensitivity (DTH) specific to the peptide in the unimmunized group.

In the results of LAIT, the LAIR of the group containing HSA, HSA-STF, and PHA was significantly higher than the other groups. This proved that, in the presence of HSA, transfer factor specific to HSA could significantly enhance the immunological activity of the lymphocyte. Moreover, it also raised the capability of specific response to differential immunogen. Thus, the data of this study demonstrated that HSA-STF possessed antigen specific activity, which depended on the antigen and the capacity of combining with the specific antigens [24].

Specific transfer factor plays an important role in regulating the immune response. A study by Pineda et al., 2005, [10] determined the role of STF in regulating the immune response using the mouse model of pleomorphism malignancy glioma and revealed that STF increases the number of CD2, CD4, CD8, and NK cells; it enhances the apoptosis of number of tumor cells and the expression of Th1 subpopulation in tumor tissue. Another study by Fujisawa et al. 1991 reported that, through a possible mechanism of STF, it could affect peripheral blood mononuclear cells, raise the production of leukocyte movement inhibition factor, promote the quantity of $\gamma$-IFN, IL-1, CD4, and CD3, and also could stimulate the expression of IL-2R [25]. The findings from a study by Fujisawa et al. 1991 [25] showed that the contents of IL-4, $\gamma$-IFN, and IL-21 were detected as the key products of T helper 1 cells (Th1). It also revealed that $\gamma$-IFN concentration was significantly higher than the lymphocytes without stimulation by HSA-STF. These results were in line with another study by Kirkpatrick et al. 1970 [26]; however, the level of IL-4 was not affected by HSA-STF. This indicated that TF specific to HSA had no effect on T helper 2 cells (Th2). These results demonstrate that HSA-STF can increase the ratio of Th1/Th2 by increasing the expression of Th1, and by relatively decreasing the Th2 clonal activity. The results of this study also demonstrate that HSA-STF could enhance cellular immunity while decreasing the humoral immunological response. Further, this study revealed that the concentration of IL-21 was the same as that of $\gamma$-IFN, but it was significantly higher than the lymphocytes without stimulation by HSA-STF. In line with our results [27, 28] Batten et al. (2010) and Eto et al. (2011) reported that IL-21 is a key cytokine secreted by $\mathrm{T}$ follicular helper cells (Tfh), it promotes the activity of Tfh and also mediates the secretion function of B cells. On the other hand, cytokines have the ability to directly affect B cells through combining IL-21R expressed on the B-cell membrane, and, thus, this helps to increase the levels of immunoglobulin secreted, which in turn enhances the humoral immunity. Thus, the results from the current study demonstrate that HSA-STF plays an important role in transferring cellular immunity to the receiver, and it also has the ability to mediate humoral immunological response.

\section{Conclusion}

In conclusion, we were successful in preparing the HSA-STF vaccine. Its physicochemical properties were evaluated, and these were in accordance with the Chinese standard of STF; HSA-STF had immunological activity which could transfer the immune response specific to HAS, and thus it could 
prove to be a potential candidate for the development of male immunocontraceptive agents. Also, because of its antigen specificity, HSA-STF probably has the abilities to enhance the effect of potential pregnancy vaccine specific to the HSA. Further, there is a need to conduct more robust studies to further corroborate our findings.

\section{Abbreviations}

STF: $\quad$ Specific transfer factor

HSA: Human sperm antigen

LPT: Lymphocyte proliferation test

LAIT: Leukocyte adhesion inhibition test

IgSF: Immunoglobulin superfamily

HSV-1: Herpes simplex virus type 1

PHA: Phytohemagglutinin

MTT: Methyl thiazolyl tetrazolium.

\section{Ethical Approval}

Human semen samples were obtained from the Medical Reproduction Centre of the Affiliated Hospital of Jining Medical College, China, and the collection was approved by the Hospital Ethics Committee. After receiving the written and signed informed consent form, the human sperm samples were collected from healthy male donors under sterile conditions.

\section{Conflict of Interests}

There is no conflict of interests for any of the authors.

\section{Acknowledgments}

The authors would like to acknowledge all the volunteers who provided the sperm samples, and the medical personnel for supporting this study. The work on sperm antigens was supported by the Nature Science Foundation of Shandong Province (no. ZR2012HL29), the High School Science and Technology Plan Program of Shandong Province (no. J11LF18), the Population and Family Planning Commission of Shandong Province (no. (2011) 13), and the Development Plan Project of Jining Science and Technology Bureau (no. (2011) 57).

\section{References}

[1] Q.-Y. Zhu, X.-J. Gu, J. Yang, J.-H. Wang, B. Tang, and H.-F. $\mathrm{Wu}$, "Purification and characterization of biologically active recombinant human Eppin expressed in Escherichia coli," Chinese Medical Journal, vol. 121, no. 7, pp. 620-624, 2008.

[2] C. E. Hoesl, F. Saad, M. Pöppel, and J. E. Altwein, "Reversible, non-barrier male contraception: status and prospects," European Urology, vol. 48, no. 5, pp. 712-723, 2005.

[3] B. E. Kurth, L. Digilio, P. Snow et al., "Immunogenicity of a multi-component recombinant human acrosomal protein vaccine in female Macaca fascicularis," Journal of Reproductive Immunology, vol. 77, no. 2, pp. 126-141, 2008.
[4] R. K. Naz, "Immunocontraceptive effect of Izumo and enhancement by combination vaccination," Molecular Reproduction and Development, vol. 75, no. 2, pp. 336-344, 2008.

[5] M. G. O'Rand, E. E. Widgren, Z. Wang, and R. T. Richardson, "Eppin: an epididymal protease inhibitor and a target for male contraception," Society of Reproduction and Fertility Supplement, vol. 63, pp. 445-453, 2007.

[6] Z. Wang, E. E. Widgren, R. T. Richardson, and M. G. O’Rand, "Characterization of an eppin protein complex from human semen and spermatozoa," Biology of Reproduction, vol. 77, no. 3, pp. 476-484, 2007.

[7] G. Pizza, D. Viza, C. De Vinci et al., "Orally administered HSVspecific transfer factor (TF) prevents genital or labial herpes relapses," Biotherapy, vol. 9, no. 1-3, pp. 67-72, 1996.

[8] J. Neequaye, D. Viza, G. Pizza, P. H. Levine, C. De Vinci, and D. V. Ablashi, "Epstein-Barr virus (EBV)-specific transfer factor," Anticancer Research, vol. 10, pp. 1183-1187, 1990.

[9] T. A. Liubchenko, O. H. Holeva, L. S. Kholodna, V. A. Stepanchuk, and A. I. Vershyhora, "Human specific transfer factor to Staphylococcus aureus antigens," Fiziolohichnyı̆ Zhurnal, vol. 43, no. 3-4, pp. 25-32, 1997 (Ukrainian).

[10] B. Pineda, S. Estrada-Parra, B. Pedraza-Medina, A. RodriguezRopon, R. Pérez, and O. Arrieta, "Interstitial transfer factor as adjuvant immunotherapy for experimental glioma," Journal of Experimental and Clinical Cancer Research, vol. 24, no. 4, pp. 575-583, 2005.

[11] G. Pizza, F. Chiodo, V. Colangeli et al., "Preliminary observations using HTV-specific transfer factor in AIDS," Biotherapy, vol. 9, no. 1-3, pp. 41-47, 1996.

[12] S. Estrada-Parra, A. Nagaya, E. Serrano et al., "Comparative study of transfer factor and acyclovir in the treatment of herpes zoster," International Journal of Immunopharmacology, vol. 20, no. 10, pp. 521-535, 1998.

[13] M. Masi, C. De Vinci, and O. R. Baricordi, "Transfer factor in chronic mucocutaneous candidiasis," Biotherapy, vol. 9, no. 1-3, pp. 97-103, 1996.

[14] T. T. Orozco, M. O. Solano, G. F. Sandoval, J. G. Vera, and S. E. Parra, "Inflammatory cells in patients with atopic dermatitis after treatment with transfer factor," Revista Alergia Mexico, vol. 51, no. 4, pp. 151-154, 2004.

[15] J. Zhou, J. Luo, W. Sun, X. Tang, and N. Qin, “The preparation a specific trasfer factor of paratyphoid A with two methods of in vivo and in vitro," Jiangsu Medical Journal, vol. 35, pp. 686-688, 2009.

[16] J. W. Zhou, J. M. Luo, W. B. Sun, R. Ma, and D. Tian, "Preparation and standardization of paratyphi a specific transfer factor," Journal of Xi'An Jiaotong University, vol. 31, no. 5, pp. 637-639, 2010.

[17] J. Zhou and J. Luo, "Current research status and clinical application of specific transfer factor," International Journal of Biologicals, vol. 32, pp. 97-100, 2009.

[18] H. Liu, R. Zhang, Y. Wu, Y. Wang, and H. Che, "Determination of free amino acids in transfer factor capsules by pre-column derivatization with RP-HPLC," Chinese Journal of Biochemical Pharmaceutics, vol. 28, pp. 233-235, 2007 (Chinese).

[19] G. Wang, C. Zhao, L. Wang, and P. Zhang, "Basic research on physicochemical properties of three transfer factors," Journal of Jilin University, vol. 16, pp. 432-435, 1990.

[20] C. H. Kirkpatrick, "Transfer factors: identification of conserved sequences in transfer factor molecules," Molecular Medicine, vol. 6, no. 4, pp. 332-341, 2000 (Chinese). 
[21] H. S. Lawrence, Transfer Factor in Cellular Immunity, vol. 68 of Harvey lecture series, Academic Press, New York, NY, USA, 1974.

[22] L. Xiao, F. Xia, and S. W. Liu, "Effect of different concentration TFtb on lymphocyte proliferation," Chinese Journal of Cellular and Molecular Immunology, vol. 20, pp. 241-242, 2004.

[23] C. H. Kirkpatrick, S. J. Rozzo, J. J. Mascali, and C. F. Merryman, "Murine transfer factor. II. Transfer of delayed hypersensitivity to synthetic antigens," Journal of Immunology, vol. 134, no. 3, pp. 1723-1727, 1985.

[24] B. Rummer and L. G. Foster, Immune Regulators in Transfer Factor, vol. 27, Academic Press, New York, NY, USA, 1979.

[25] T. Fujisawa, S. Sasakawa, Y. Likura, F. Komatsu, and Y. Yamaguchi, Recent Advances in Transfer Factor and Dialyzable Leucocyte Extracts, Maruzen Press, Tokyo, Japan, 1991.

[26] C. H. Kirkpatrick, J. W. Chandler, and R. N. Schimke, "Chronic mucocutaneous moniliasis with impaired delayed hypersensitivity," Clinical and Experimental Immunology, vol. 6, no. 3, pp. 375-385, 1970.

[27] M. Batten, N. Ramamoorthi, N. M. Kljavin et al., "IL-27 supports germinal center function by enhancing IL-21 production and the function of T follicular helper cells," Journal of Experimental Medicine, vol. 207, no. 13, pp. 2895-2906, 2010.

[28] D. Eto, C. Lao, D. DiToro et al., "IL-21 and IL-6 are critical for different aspects of $\mathrm{B}$ cell immunity and redundantly induce optimal follicular helper CD4 T cell (Tfh) differentiation," PLoS One, vol. 6, no. 3, Article ID e17739, 2011. 


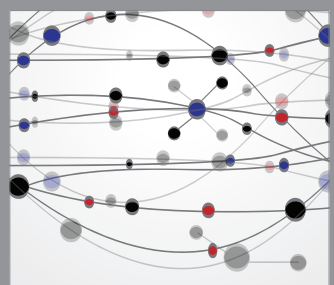

The Scientific World Journal
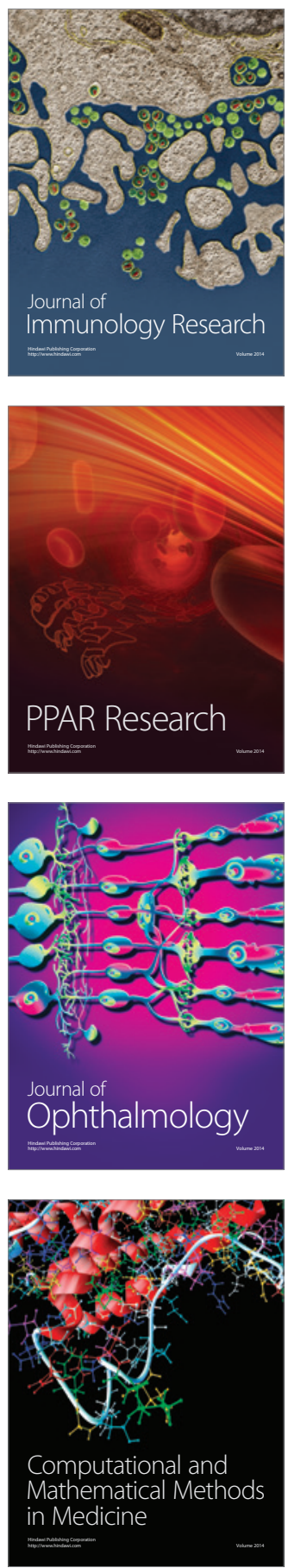

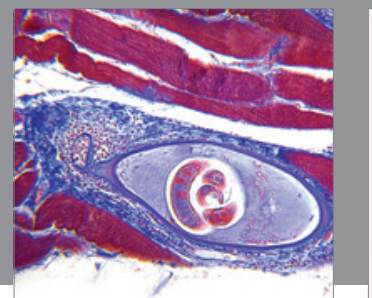

Gastroenterology

Research and Practice
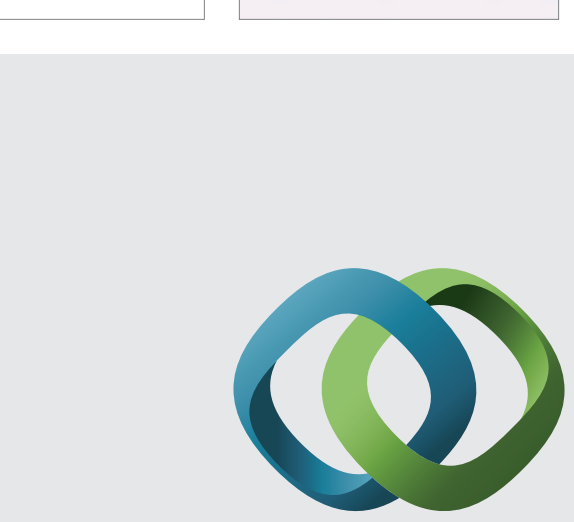

\section{Hindawi}

Submit your manuscripts at

http://www.hindawi.com
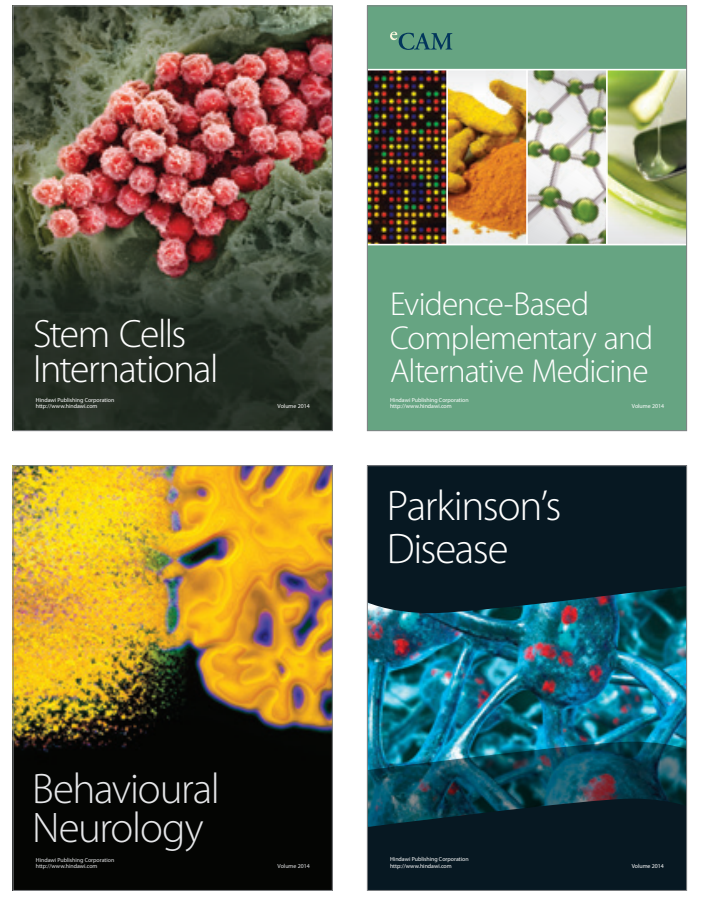
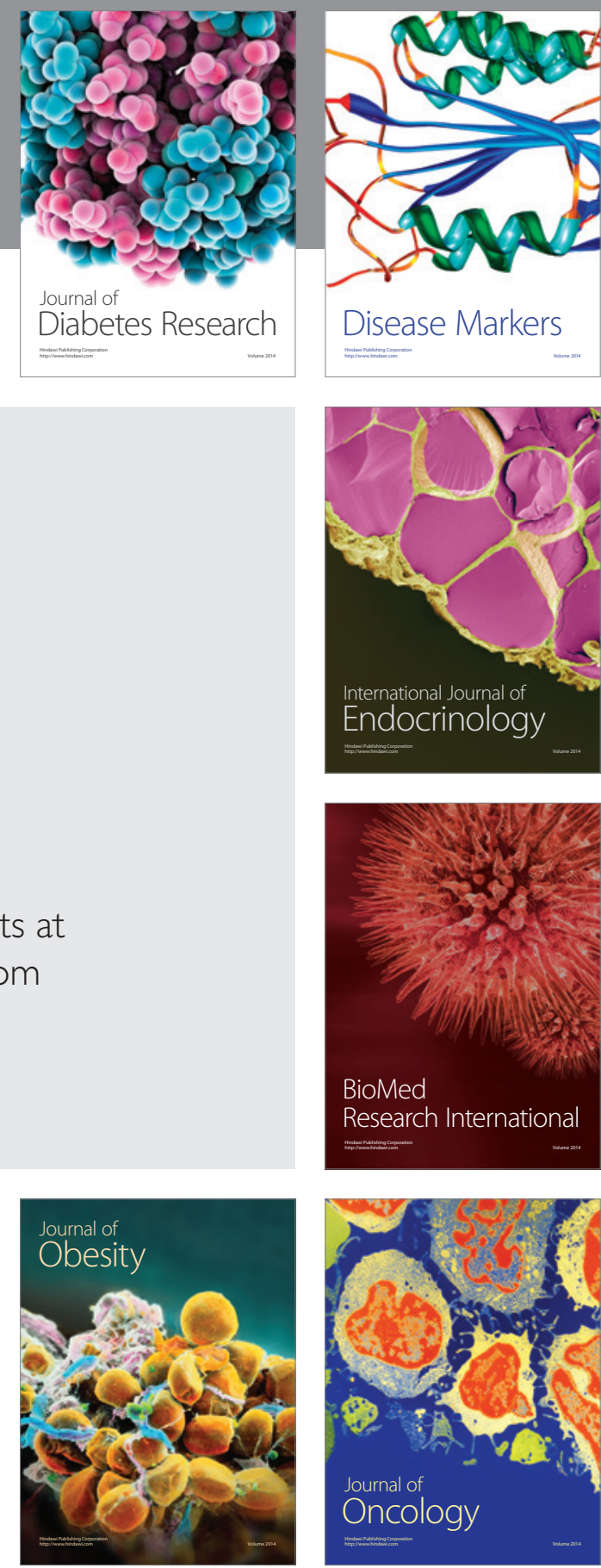

Disease Markers
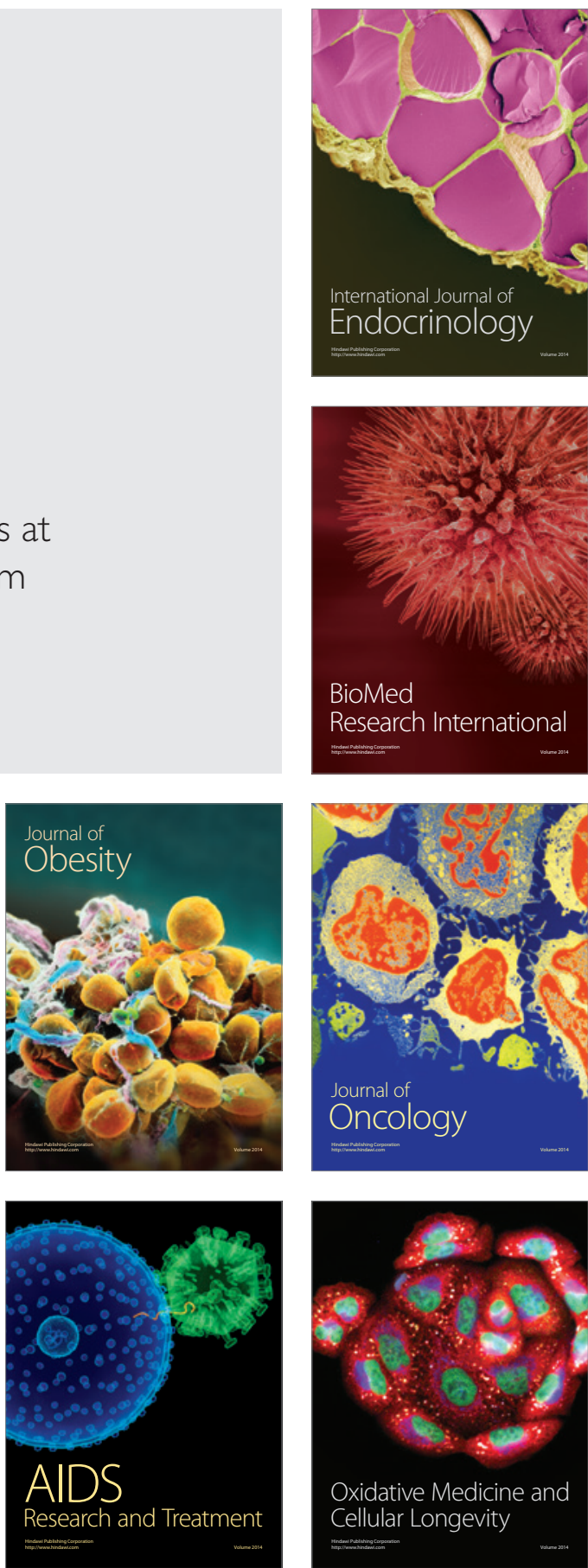